\title{
Clinical trial for uniform multidrug therapy for leprosy patients in Brazil (U-MDT/CT-BR): adverse effects approach ${ }^{*}$
}

\author{
Rossilene Conceição da Silva Cruz ${ }^{1,11}$ \\ Gerson Oliveira Penna ${ }^{3,4}$ \\ Heitor de Sá Gonçalves ${ }^{7}$ \\ Maria Lúcia Fernandes Penna ${ }^{9}$ \\ Sinésio Talhari ${ }^{10,11}$
}

\author{
Samira Bührer-Sékula ${ }^{2,11}$ \\ Maria Elisabete Amaral de Moraes ${ }^{5,6}$ \\ Mariane Martins de Araújo Stefani ${ }^{8}$ \\ Maria Araci de Andrade Pontes ${ }^{7}$
}

DOI: http:/ / dx.doi.org/10.1590/abd1806-4841.20186709

\begin{abstract}
BACKGRound: The Clinical Trial for Uniform Multidrug Therapy for Leprosy Patients in Brazil (U-MDT/CT-BR), designed to evaluate the effectiveness of a six-months regimen, assessed the adverse effects caused by the drugs.

ОвјестіVE: Describe adverse effects due to MDT in U-MDT/CT-BR, comparing the uniform regimen (U-MDT) to the current WHO regimen (R-MDT). Patients and methods: After operational classification, patients were randomly allocated to the study groups. U-MDT PB and U-MDT MB groups, received the U-MDT regimen, six doses of MB-MDT (rifampicin, dapsone and clofazimine). R-MDT PB and R-MDT MB groups, received the WHO regimens: six doses (rifampicin and dapsone) for PB and 12 doses (rifampicin, dapsone and clofazimine) for MB. During treatment, patients returned monthly for clinical and laboratorial evaluation. Patients with single lesion were not included in this trial.

RESULTS: Skin pigmentation $(21.7 \%)$ and xerosis $(16.9 \%)$ were the most frequent complaints among 753 patients. Laboratory exams showed hemoglobin concentration lower than $10 \mathrm{~g} / \mathrm{dL}$ in $23.3 \%$ of the patients, glutamic oxaloacetic transaminase (GOT) above $40 \mathrm{U} / \mathrm{L}$ in $29.5 \%$ and glutamic pyruvic transaminase (GPT) above $40 \mathrm{U} / \mathrm{L}$ in $28.5 \%$. Twenty-four patients (3.2\%) stopped dapsone intake due to adverse effects, of whom $16.6 \%$ due to severe anemia. One case of sulfone syndrome was reported. STUDY LIMITATIONS: Loss of some monthly laboratory sample collection.

CONCLUSIONS: There was no statistical difference regarding adverse effects in the R-MDT and U-MDT groups but anemia was greater in patients from R-MDT/MB group, therefore adverse effects do not represent a constraint to recommend the sixmonth uniform regimen of treatment for all leprosy patients.
\end{abstract}

Keywords: Abnormalities, drug-induced; Drug monitoring; Leprosy; Therapeutics

\section{INTRODUCTION}

Leprosy is a neglected tropical disease, and remains a public health problem in many countries, including Brazil. The diagnosis is mainly clinical. The current treatment, multidrug therapy (MDT), recommended by WHO in 1981, is based on the combination of drugs: dapsone and rifampin for patients classified as paucibacillary $(\mathrm{PB})$, and dapsone, rifampicin and clofazimine for multibacillary patients (MB). ${ }^{1}$ Since 1982 , millions of patients were treated and cured with MDT and report of adverse effects (AE) is considerably low when compared to the benefit for patients and Leprosy Control Programs. ${ }^{2}$

\footnotetext{
Received 17 November 2016.

Accepted 15 May 2017.

* Work conducted at Centro de Dermatologia Dona Libânia and Fundação de Dermatologia Tropical e Venereologia, "Alfredo da Matta" (FUAM), Manaus (AM), Brazil.

Financial support: Departamento de Ciência e Tecnologia (DECIT), Ministério da Saúde CNPq, Conselho Nacional de Desenvolvimento Científico e Tecnológico Conflict of interest: None.

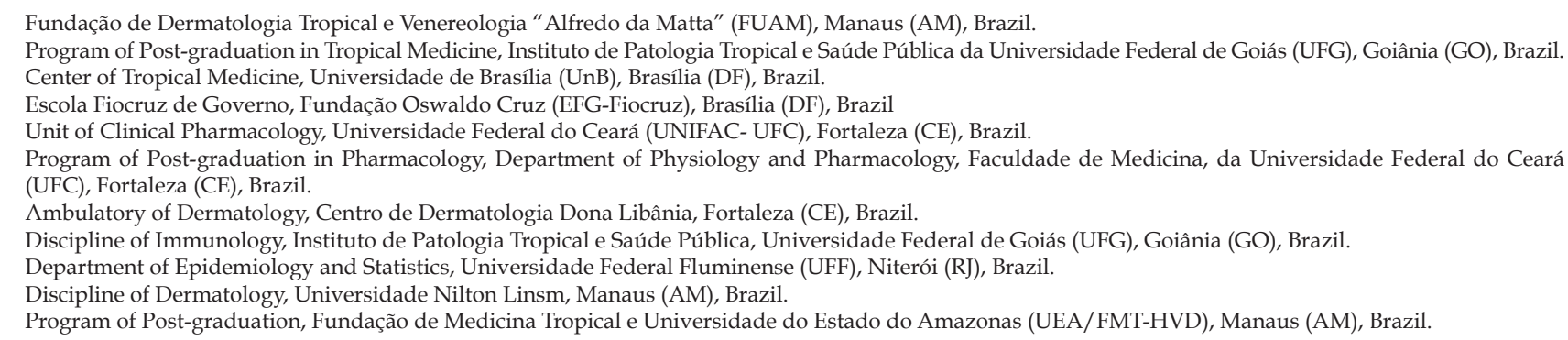


However, AE caused by these drugs can be severe and discontinuation of treatment should be necessary in some patients. Dapsone alone can cause methemoglobinemia, hemolytic anemia and other haematological disorders. Hepatitis, pancreatitis, and renal involvement may occur.

Although severe AE are rare, there are reports of several different AE. ${ }^{1}$ A retrospective study in India, with $10,426 \mathrm{MB}$ patients and 35,013 PB patients, reported 24 cases of severe $\mathrm{AE}$, resulting in treatment discontinuation. ${ }^{3}$ A prospective study, including 176 patients, reported $45 \%$ AE. ${ }^{4}$ Retrospective studies with MDT in Brazil, reported $15 \%$ and $45 \%$ of AE. ${ }^{5,6}$ Dermatological problems, toxic hepatitis, hemolytic anemia, renal failure, and thrombocytopenia were the main cause of MDT discontinuation in other Brazilian studies. ${ }^{7,8}$ Another retrospective study found $19 \%$ of $\mathrm{AE} ; 49 \%$ was related to hematological causes, and $50 \%$ to liver function. ${ }^{9}$

In order to implement leprosy control in the field, WHO has made changes in the treatment duration. The number of doses for MB patients has been the main concern: the first guideline, in 1981, recommended 24 doses of MDT or, if possible, to treat until slit skin smears negativity. ${ }^{1}$ Subsequently, 24 doses was recommended as a fixed duration treatment, and in 1998, WHO recommended 12 doses for MB patients. ${ }^{10}$ Despite the MDT success, the long treatment duration, the difficulty to implement treatment, and the low adherence for treatment, reinforces the need for shorter treatment regimen. ${ }^{11,12}$ In 2002, the WHO Leprosy Expert Committee recommended pilot trials to evaluate the possibility of a uniform MDT regimen (U-MDT) with 6 doses of MDT-MB for all leprosy patients, independently of any classification. ${ }^{13}$

A multicenter clinical trial to assess the effectiveness of a U-MDT regimen was initiated in India and China in $2004 .{ }^{14} \mathrm{Up}$ to now, clinical improvement of PB patients, and good acceptability of MB patients are the main aspects observed. Moreover, the regimen was easier to implement in general health services. Another study conducted in India, concluded that the U-MDT was effective for treatment of $\mathrm{PB}$ patients but the follow up was not adequate to evaluate the efficacy for MB patients and it was not compared with MDT/ WHO. ${ }^{15}$ No increase of relapses among MB patients treated with U-MDT was observed in a recent study conducted in Bangladesh. ${ }^{16}$

To evaluate the effectiveness of the new treatment regimen (U-MDT) for leprosy patients, an open-label, randomized and controlled clinical trial - (Clinical Trial for Uniform Multidrug Therapy for Leprosy Patients in Brazil (U-MDT/CT-BR) - was conducted in two Brazilian national referral centers of the National Leprosy Control Programme: Alfredo da Matta Foundation (FUAM) in Manaus, state of Amazonas, and Dona Libânia Dermatology Centre (CDERM), in Fortaleza, state of Ceará. The efficacy of the treatment regimens, decrease of bacteriological index, frequency of reactions, and acceptability of U-MDT for PB patients due to clofazimine, were the main objectives of the study.

Here, we compare the main adverse effects observed in the different groups and treatment regimens.

\section{METHODS}

A total of 753 patients were recruited from March 2007 to February 2012, at two National Brazilian referral leprosy centers:
Dona Libânia (Fortaleza, Ceará) and Alfredo da Matta (Manaus, Amazonas). Patients were classified as PB and MB according to the number of skin lesions and randomly assigned to U-MDT or R-MDT sub-groups. Further protocol details may be found in "Clinical Trial for Uniform Multidrug Therapy for Leprosy Patients in Brazil (U-MDT/CT-BR): Rationale and Design" with open-access at the site. ${ }^{17}$

The patients returned monthly for medical evaluation and to receive blister packs. Full blood count (RBC - red blood cells, $\mathrm{Hb}$ - hemoglobin, HT- hematocrit, MCHC - mean concentration hemoglobin corpuscular, $\mathrm{MCH}$ - mean corpuscular hemoglobin, $\mathrm{MCV}$ mean corpuscular volume, and liver function tests - GOT and GPT (glutamic oxaloacetic transaminase and glutamic pyruvic transaminase) were done before MDT, and monthly, during treatment. Biochemical urea, creatinine, alkaline phosphatase, and bilirubin were performed before treatment, and whenever necessary.

During monthly visits, patients were examined and asked about leprosy reactions and adverse effects; this information was registered on the Clinical Registration Forms (CRF). Patients were encouraged to return at any time in case of any abnormality. The main adverse effects were characterized as follows: Anemia - decrease of hemoglobin from baseline (less than $10 \mathrm{~g} / \mathrm{dL}$ ), and the possibility of association with fatigue, dyspnea, dizziness and jaundice; methemoglobinemia - presence of cyanosis, dyspnea, dizziness, headache and/or fatigue; psychiatric disorders - depression, psychotic or anxiety behavior; xerosis - dryness of the skin, with or without pruritus and scaling; skin pigmentation: darkness of the skin; erythroderma - erythematous eruption extending to over $90 \%$ of the body surface; urticaria - edematous itchy plaques, with or without angioedema; dapsone syndrome - exfoliative dermatitis, accompanied by fever, hepatosplenomegaly and liver functions abnormalities; flu-like syndrome - fever, runny nose, sore throat, cough, nausea, vomiting, poor appetite, headache, muscle/joint aches, and malaise; hepatotoxicity, mild - serum aminotransferases levels from $40 \mathrm{IU} / 1$ to $100 \mathrm{IU} / 1$; moderate to severe - aminotransferases over $100 \mathrm{IU} / 1$, with or without clinical evidence of jaundice, malaise and other symptom. The criteria for mild, moderate or severe for each side-effect were based on the U.S. National Cancer Institute - Cancer Therapy Evaluation Program, Common Terminology Criteria for Adverse Events, version 3.0, see. Protocolo MDTU-Rationale and Design - Supplementary data 1, with open-access at the site http://memorias.ioc.fiocruz.br or at http://www.scielo/mioc. ${ }^{17,18}$

Patients randomized to the groups PB/U-MDT and PB/RMDT, receiving 6 MDT doses, returned 30 days after the last dose for the first evaluation after MDT completion. Patients randomized to MB/U-MDT group, receiving 6 MDT doses, were closely monitored and evaluated twelve times as the MB/R-MDT patients that received 12 MDT doses. Therefore, the $13^{\text {th }}$ blood collection corresponds to 210 days after the last MDT dose for MB/U-MDT patients and 30 days for MB/R-MDT patients.

\section{Ethical considerations}

The study was performed under the International (Helsinki) and Brazilian research regulations involving humans and was approved on the $17^{\text {th }}$ February 2006, (protocol 001/06) by the ethical 
committees of the two institutions involved in the research and the National Ethics Commission. Written informed consent was obtained from all the patients. Written parental consent was obtained for patients with 6 to 17 years old. Data confidentiality was guaranteed and the patients were free to leave the study at any time during the trial (ClinicalTrials.gov identifier - NCT00669643).

\section{Statistical analysis}

The following hematological values were used as normal parameters: red blood cells - minimum of 4.4 million cells for male and 4.2 million for female; hemoglobin $-13 \mathrm{~g} / \mathrm{dL}$ for male and $12 \mathrm{~g} /$ dL for female; hematocrit $-43 \%$ for male and $37 \%$ for female; leukocytes: 3,600 uL, MCHC: 30 - 35g/dL (\%), MCV: 80 - 95 fL and MCH: 27 - 34 pg. ${ }^{18,19}$

Data are expressed as mean \pm SD. Analysis of Variance (ANOVA test was used for testing the significance of parametric data using SPSS, KAPLAN-MEIER test was used for analytical risk of anaemia, taken as the cut off value for significance as the limit for anemia was considered less or equal 10 for hemoglobin.

\section{RESULTS}

A total of 753 patients were enrolled in the study. In table 1 are presented the main baseline characteristics: $59.5 \%$ (448) were male; $80.3 \%$ (605) were 20 to 59 years old. The gender distribution was different in the two WHO operational classification groups, females were $67.3 \%$ (105/159) in the PB group, and MB were $33.6 \%$ (200/594). Regarding the classification of the patients, $78.9 \%$ (594) were multibacillary, and $47.4 \%$ (282/594) had bacterial index (BI) equal or higher than 3 .

A total of 10,600 adverse effects (AE) were registered. The most frequent $\mathrm{AE}$ were skin pigmentation and xerosis: 2301 (21.7\%) and 1787 (16.9\%) patients, respectively. For all the other symptoms and signs there was less than $5 \%$ of AE. Concerning skin pigmentation, it varied from mild, in 1715 (16.2\%), to moderate in 297 (5.4\%), and severe in $14(0.1 \%)$ patients. Mild, moderate or severe xerosis was observed in 1444 (13.6\%), 297 (2.8\%) and $46(0.4 \%)$, respectively. The main AE are presented in table 2.

During treatment, $253(29.5 \%)$ and 244 (28.5\%) patients had changes (>40) in GOT and GPT values, without statistical significance. GTP and GOT were above 100 at any time during the treatment, in 18 and 36 patients, respectively. However, even in these patients, it was not necessary to interrupt the treatment.

There was no statistical significant difference among the study groups for GOT and GPT variation during treatment (Figure 1).

Severe $\mathrm{AE}$ were observed in $0.1 \%$ of all patients. The main hematological variation was anemia $(\mathrm{Hb}<10 \mathrm{~g} / \mathrm{dl})$; it was observed in $201(26.7 \%)$ patients but there was no significant difference between the U-MDT and the R-MDT groups $(p=0.2242)$. With the exception of leukocytes and MCV hematological parameters, all others presented lower average for the PB/U-MDT compared to other groups. The hematological averages were higher for MB/U-MDT group, receiving 6 doses, than the MB/R-MDT, receiving 12 doses of treatment regimen.

Regardless of gender there was a decrease in the mean hemoglobin level during treatment in all arms of the study, particularly a month after the first MDT dose, with a gradual recover, $\mathrm{p}<0.0000$. The decrease was statistically significant among the study groups (Figure 2). A recover is observed for all groups but is more evident for males. MB/RMDT patients receiving longer treatment, the basal hemoglobin value was not achieved 30 days after treatment completion. Similarly, erythrocytes and hematocrit showed a decrease throughout the period of treatment, particularly a month after first dose in all groups. The difference among experimental and regular treatment groups was statistically significant.

\begin{tabular}{|c|c|c|c|c|c|c|c|c|c|}
\hline \multirow{2}{*}{$\begin{array}{l}\text { Characteristics } \\
\text { Mean age / Age group }\end{array}$} & \multicolumn{4}{|c|}{ Paucibacillary } & \multicolumn{4}{|c|}{ Multibacillary } & \multirow{2}{*}{ TOTAL } \\
\hline & \multicolumn{2}{|c|}{ U-MDT 41.44} & \multicolumn{2}{|c|}{ R-MDT 36.08} & \multicolumn{2}{|c|}{ U-MDT 42.16} & \multicolumn{2}{|c|}{ R-MDT 41.44} & \\
\hline $0-9$ & 1 & $1.3 \%$ & 2 & $2.4 \%$ & 5 & $1.6 \%$ & 6 & $2.2 \%$ & $14(1.9 \%)$ \\
\hline $10-19$ & 9 & $11.7 \%$ & 17 & $20.7 \%$ & 25 & $7.8 \%$ & 24 & $8.8 \%$ & $75(10.0 \%)$ \\
\hline $20-29$ & 12 & $15.6 \%$ & 13 & $15.9 \%$ & 65 & $20.2 \%$ & 45 & $16.5 \%$ & 135 (17.9\%) \\
\hline $30-39$ & 21 & $27.3 \%$ & 18 & $22.0 \%$ & 59 & $18.4 \%$ & 52 & $19.0 \%$ & 150 (19.9\%) \\
\hline $40-49$ & 11 & $14.3 \%$ & 19 & $23.2 \%$ & 68 & $21.2 \%$ & 63 & $23.1 \%$ & $161(21.4 \%)$ \\
\hline $50-59$ & 18 & $23.4 \%$ & 10 & $12.2 \%$ & 68 & $21.2 \%$ & 63 & $23.1 \%$ & 159 (21.1\%) \\
\hline $60-69$ & 5 & $6.5 \%$ & 3 & $3.7 \%$ & 31 & $9.7 \%$ & 20 & $7.3 \%$ & $59(7.8 \%)$ \\
\hline \multicolumn{10}{|l|}{ Sex } \\
\hline Male & 25 & $32.5 \%$ & 29 & $35.4 \%$ & 215 & $67.0 \%$ & 179 & $65.6 \%$ & 448 (59.5\%) \\
\hline Female & 52 & $67.5 \%$ & 53 & $64.6 \%$ & 106 & $33.0 \%$ & 94 & $34.4 \%$ & 305 (40.5\%) \\
\hline \multicolumn{10}{|l|}{ IB } \\
\hline 0 & 71 & $92.2 \%$ & 80 & $97.6 \%$ & 96 & $29.9 \%$ & 80 & $29.3 \%$ & $327(43.4 \%)$ \\
\hline $0.1-2.99$ & $6^{*}$ & $7.8 \%$ & $2^{*}$ & $2.4 \%$ & 75 & $23.4 \%$ & 61 & $22.3 \%$ & 144 (19.1\%) \\
\hline$>3$ & 0 & $0 \%$ & 0 & $0 \%$ & 150 & $46.7 \%$ & 132 & $48.4 \%$ & $282(37.5 \%)$ \\
\hline Total & 77 & $10.2 \%$ & 82 & $10.9 \%$ & 321 & $42.6 \%$ & 273 & $36.3 \%$ & 753 \\
\hline
\end{tabular}

*For treatment purpose, recruited patients were classified based on number of skin lesions, up to five lesions as PB and or mores lesions as MB. 


\begin{tabular}{|c|c|c|c|c|c|c|c|c|c|c|}
\hline \multirow{2}{*}{ Signs and Symptoms } & \multicolumn{2}{|c|}{ Absent } & \multicolumn{2}{|c|}{ Mild } & \multicolumn{2}{|c|}{ Moderate } & \multicolumn{2}{|c|}{ Severe } & \multicolumn{2}{|c|}{ Total } \\
\hline & $\mathbf{N}$ & $\%$ & $\mathbf{N}$ & $\%$ & $\mathbf{N}$ & $\%$ & $\mathbf{N}$ & $\%$ & $\mathbf{N}$ & $\%$ \\
\hline Skin pigmentation & 8298 & $78.3 \%$ & 1715 & $16.2 \%$ & 572 & $5.4 \%$ & 14 & $0.1 \%$ & 10599 & $100 \%$ \\
\hline Xerosis & 8813 & $83.1 \%$ & 1444 & $13.6 \%$ & 297 & $2.8 \%$ & 46 & $0.4 \%$ & 10600 & $100 \%$ \\
\hline Itch & 10252 & $96.7 \%$ & 280 & $2.6 \%$ & 56 & $0.5 \%$ & 10 & $0.1 \%$ & 10598 & $100 \%$ \\
\hline Headache & 10278 & $97.0 \%$ & 287 & $2.7 \%$ & 34 & $0.3 \%$ & 1 & $0.0 \%$ & 10600 & $100 \%$ \\
\hline Paleness & 10286 & $97.0 \%$ & 284 & $2.7 \%$ & 27 & $0.3 \%$ & 3 & $0.0 \%$ & 10600 & $100 \%$ \\
\hline Fatigue & 10363 & $97.8 \%$ & 214 & $2.0 \%$ & 20 & $0.2 \%$ & 2 & $0.0 \%$ & 10599 & $100 \%$ \\
\hline Myalgia & 10389 & $98.0 \%$ & 184 & $1.7 \%$ & 24 & $0.2 \%$ & 3 & $0.0 \%$ & 10600 & $100 \%$ \\
\hline Anorexia & 10458 & $98.7 \%$ & 118 & $1.1 \%$ & 24 & $0.2 \%$ & 0 & $0.0 \%$ & 10600 & $100 \%$ \\
\hline Dyspnea & 10475 & $98.8 \%$ & 109 & $1.0 \%$ & 16 & $0.2 \%$ & 0 & $0.0 \%$ & 10600 & $100 \%$ \\
\hline Nausea & 10484 & $98.9 \%$ & 97 & $0.9 \%$ & 17 & $0.2 \%$ & 2 & $0.0 \%$ & 10600 & $100 \%$ \\
\hline Abdominal pain & 10485 & $98.9 \%$ & 97 & $0.9 \%$ & 13 & $0.1 \%$ & 5 & $0.0 \%$ & 10600 & $100 \%$ \\
\hline Weight loss & 10496 & $99.0 \%$ & 92 & $0.9 \%$ & 12 & $0.1 \%$ & 0 & $0.0 \%$ & 10600 & $100 \%$ \\
\hline Fever & 10499 & $99.1 \%$ & 80 & $0.8 \%$ & 20 & $0.2 \%$ & 0 & $0.0 \%$ & 10599 & $100 \%$ \\
\hline Diarrhea & 10524 & $99.3 \%$ & 61 & $0.6 \%$ & 15 & $0.1 \%$ & 0 & $0.0 \%$ & 10600 & $100 \%$ \\
\hline Skin rash & 10538 & $99.4 \%$ & 41 & $0.4 \%$ & 18 & $0.2 \%$ & 1 & $0.0 \%$ & 10598 & $100 \%$ \\
\hline Constipation & 10545 & $99.5 \%$ & 47 & $0.4 \%$ & 8 & $0.1 \%$ & 0 & $0.0 \%$ & 10600 & $100 \%$ \\
\hline Erythroderma & 10548 & $99.5 \%$ & 26 & $0.2 \%$ & 21 & $0.2 \%$ & 5 & $0.0 \%$ & 10600 & $100 \%$ \\
\hline Photodermatosis & 10552 & $99.5 \%$ & 23 & $0.2 \%$ & 24 & $0.2 \%$ & 1 & $0.0 \%$ & 10600 & $100 \%$ \\
\hline Depression & 10553 & $99.6 \%$ & 37 & $0.3 \%$ & 8 & $0.1 \%$ & 0 & $0.0 \%$ & 10598 & $100 \%$ \\
\hline Bleeding & 10562 & $99.6 \%$ & 27 & $0.3 \%$ & 11 & $0.1 \%$ & 0 & $0.0 \%$ & 10600 & $100 \%$ \\
\hline Vomiting & 10563 & $99.7 \%$ & 26 & $0.2 \%$ & 10 & $0.1 \%$ & 1 & $0.0 \%$ & 10600 & $100 \%$ \\
\hline Cyanosis & 10575 & $99.8 \%$ & 17 & $0.2 \%$ & 7 & $0.1 \%$ & 1 & $0.0 \%$ & 10600 & $100 \%$ \\
\hline Jaundice & 10579 & $99.8 \%$ & 14 & $0.1 \%$ & 7 & $0.1 \%$ & 0 & $0.0 \%$ & 10600 & $100 \%$ \\
\hline
\end{tabular}

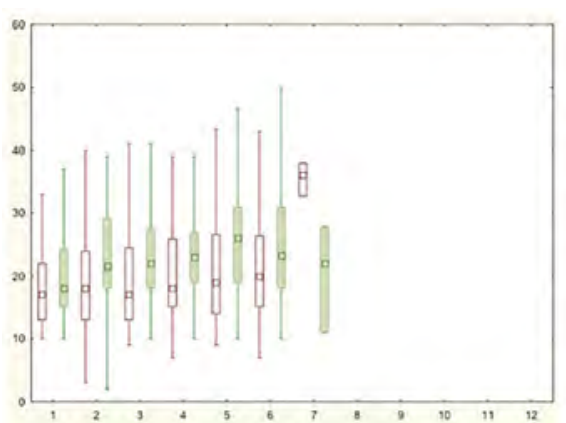

$\mathrm{PB}-\mathrm{UMDT}$

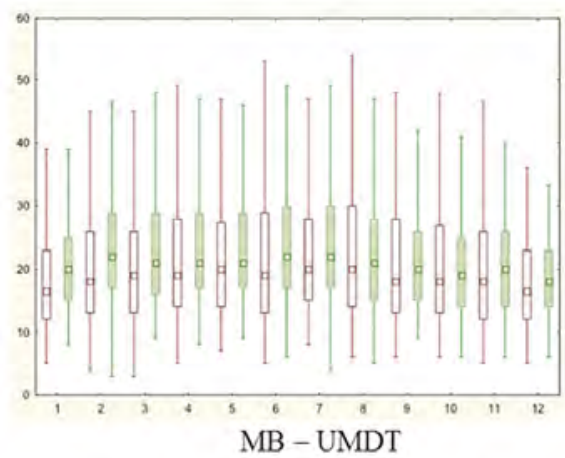

GPT PB/U-MDT - Uniform regimen Paucibacillary MB/U-MDT -Uniform regimen Multibacillary GOT 1 PB/R-MDT - Regular regimen Paucibacillary MB/R-MDT - Regular regimen Multibacillary

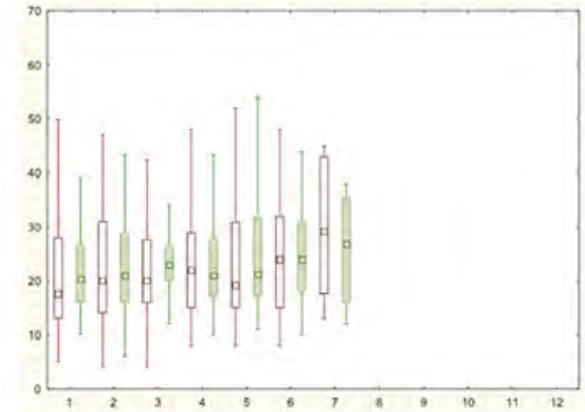

PB - RMDT

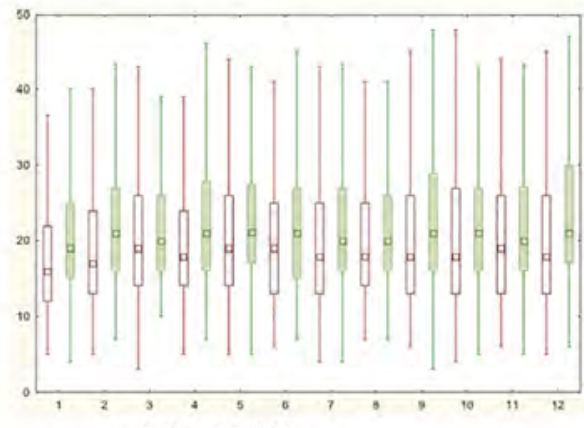

$M B$ - RMDT
FIGURE 1:

Changes in transaminases during treatment in 753 leprosy patients, according to study groups 

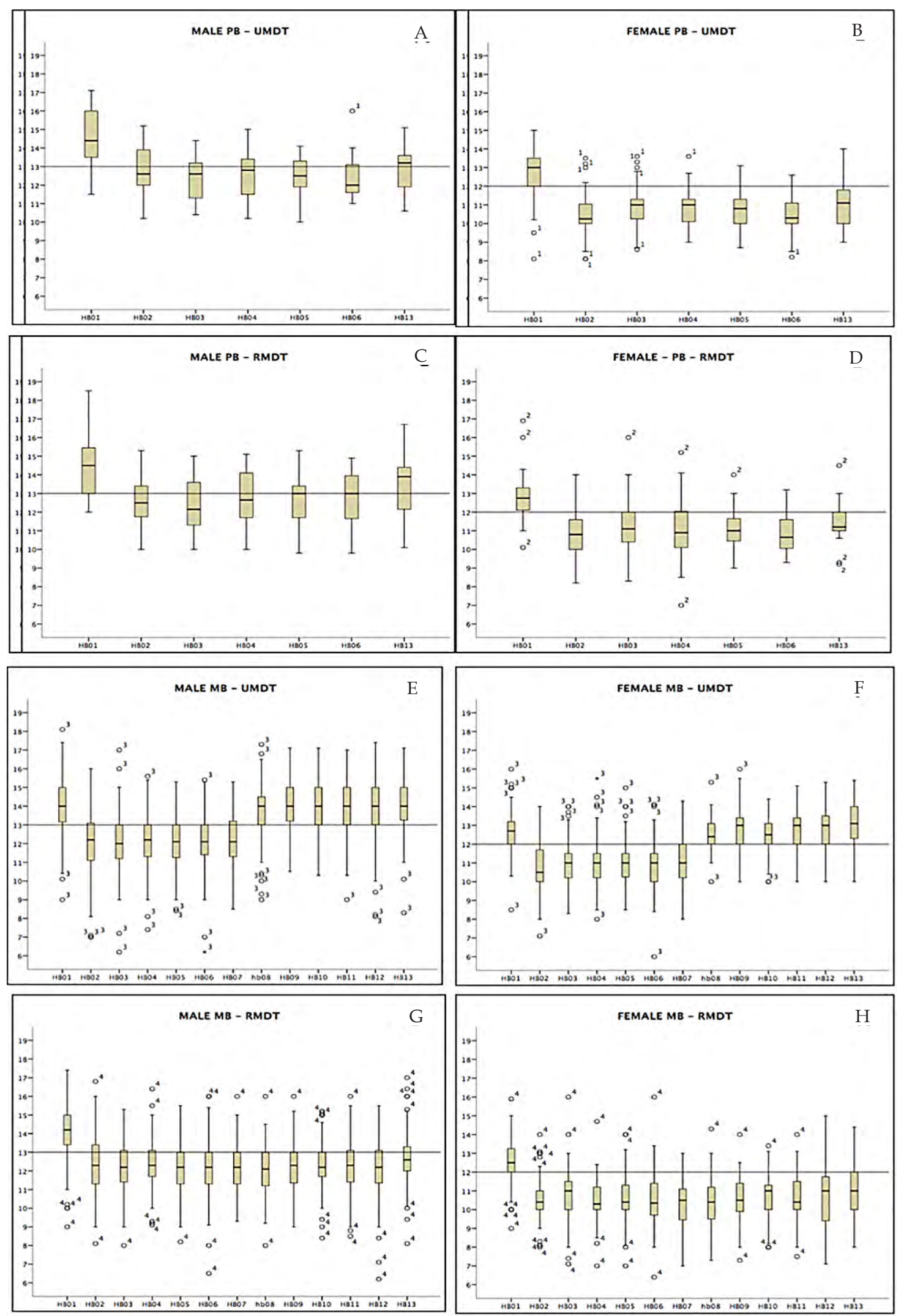

PB/U-MDT - Uniform regimen Paucibacillary $\mathrm{MB} / \mathrm{U}-\mathrm{MDT}$ - Uniform regimen Multibacillary PB/R-MDT - Regular regimen Paucibacillary $\quad \mathrm{MB} / \mathrm{R}-\mathrm{MDT}$ - Regular regimen Multibacillary Male reference line $13 \mathrm{~g} / \mathrm{dl} ;$ Female reference line $12 \mathrm{~g} / \mathrm{dl}$

FIGURE 2: Mean hemoglobin level variation during treatment of 753 patients by group and sex, MDT CT/BR 
A

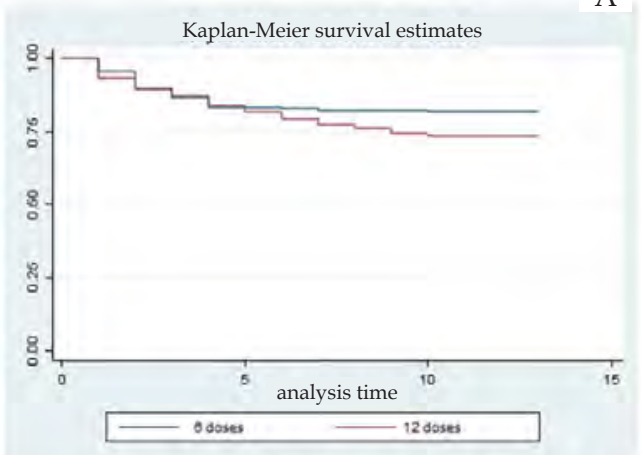

6 doses $=$ Patients from PB/UMDT, PB $/$ RMDT and MB /U-MDT; 12 doses = Patients from MB $/$-MDT
B

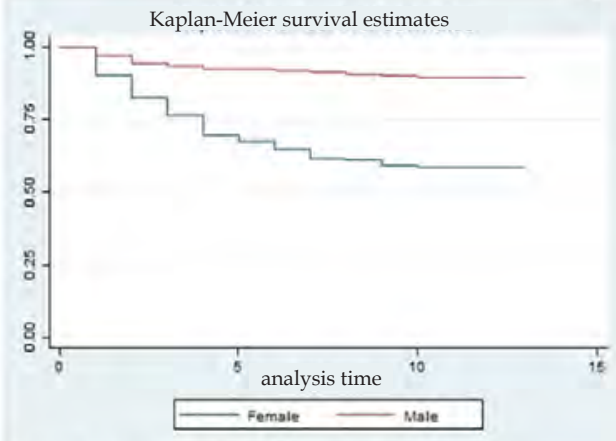

FIGURE 3: Patient's risk for anemia according to treatment duration and gender
TABLE 3: Causes of MDT discontinuation

\begin{tabular}{lcc} 
Cause & N & $\%$ \\
\hline Anemia & 16 & $66.7 \%$ \\
Erythroderma & 3 & $12.5 \%$ \\
Methemoglobinemia & 1 & $4.2 \%$ \\
Urticaria & 1 & $4.2 \%$ \\
Headache & 1 & $4.2 \%$ \\
Psychiatric disorders & 1 & $4.2 \%$ \\
Sulfone syndrome & 1 & $4.2 \%$ \\
\hline
\end{tabular}

Comparing the hemoglobin results between PB groups, no statistical difference was observed. However, a significant statistical difference was observed between MB groups $(p<0.0000)$. In all groups, female patients presented reduced levels of red blood cells, hematocrit and hemoglobin more pronounced than in males, with statistical significance - $(\mathrm{p}<0.0000)$.

Considering the other red blood cell results, it was observed that only MCHC did not show statistically significant parameters when the mean average between the different groups during treatment was compared. Although more evident in the PB/U-MDT group, $\mathrm{MCV}$ and $\mathrm{MCH}$ presented high basal mean increase after the first dose in all groups and remained high during treatment. In MB/U-MDT group, a decrease was observed only after completion of treatment, almost reaching baseline levels; the MB/R-MDT group remained high during all treatment.

Kaplan-Meier test was employed for the analysis of anemia risk related to treatment duration and gender. Comparing groups, according to treatment duration, it was observed that patients treated with 12 doses (MB/R-MDT) presented a statistically significant risk for anemia when compared to patients treated with six doses $(\mathrm{PB} / \mathrm{U}-\mathrm{MDT}+\mathrm{PB} / \mathrm{R}-\mathrm{MDT}+\mathrm{MB} / \mathrm{U}-\mathrm{MDT})$ ( $\mathrm{p}$ value 0.005 ) (Figure $3 \mathrm{~A})$. Figure $3 \mathrm{~B}$ shows that females had an increased risk for developing anemia $(\mathrm{p}<0.0000)$.

Twenty-four patients (3.2\%) stopped dapsone because of $\mathrm{AE}$ and received an alternative treatment; 16 (66.7\%) had anemia, three patients of this group had leukopenia and two developed mild increase in the aminotransferases levels. Three patients (12.5\%) developed erythroderma secondary to dapsone, and all these patients had mild anemia. Methemoglobinemia was diagnosed in one pa- tient. Other reasons for MDT interruption were urticaria, headache and psychiatric disorder (Table 3).

A ten-year-old boy was diagnosed with dapsone syndrome (Drug Reaction with Eosinophilia and Systemic Symptoms - DRESS Syndrome). He developed exfoliative dermatitis, hepatosplenomegaly, fever, malaise and dyspnea. The blood tests revealed anemia $(\mathrm{Hb}=7.2 \mathrm{~g} / \mathrm{dL})$, leukocytosis (10900), increased levels of GOT (672) and GPT (919). Dapsone was stopped, and the patient was hospitalized. Thereafter, alternative MDT was introduced.

\section{DISCUSSION}

Adverse effects are among the main problems related to leprosy treatment, and most studies are retrospective. ${ }^{5,8} \mathrm{Singh}^{4}$ et al, did one of the few prospective studies to investigate AE; 176 patients under MDT treatment were followed during two years. A total number of $79 \mathrm{AE}$ was observed. The most important AE were: gastrointestinal manifestations - 76 (96.2\%), hepatic abnormalities - 15 (10.98\%), Anemia $-13(16.5 \%)$ patients. A total of 9 (11.8\%) of patients that had to stop treatment.

The main AE presented in this paper is part of the prospective Brazilian Clinical Trial for Uniform Multidrug Therapy of Leprosy (U-MDT/CT-BR). ${ }^{17}$

Skin pigmentation and xerosis were the most frequent $\mathrm{AE}$ in all the arms of the study; they were caused by clofazimine. These findings might explain why clofazimine intake by PB patients caused no impact in patient's satisfaction, as previously reported by our group..$^{20}$

Serum aminotransferases increased levels was observed in $272(31.5 \%)$ patients. These changes could be associated with any of the different component of MDT, but rifampicin is the most frequently related drug to this AE. There was no statistical significant difference in the serum aminotransferase levels in the four arms of the study. No patient had to stop rifampicin or clofazimine.

Hematological parameters alterations occurred in all groups and statistical significance was observed between MB/U-MDT and MB/R-MDT. The faster recovery of hematological parameters observed in the MB/U-MDT group was probably because U-MDT has only six doses and there was a long period between the last MDT dose and the blood collection accomplished for all MB patients on the $13^{\text {th }}$ month after the beginning of MDT.

Red blood cells, hematocrit and hemoglobin levels analysis 
according to gender and groups of treatment, presented significant higher decrease on female patients. When Kaplan Meyer analysis was performed only for gender, a statistically significant greater risk for anemia was observed on females. Dupnik ${ }^{6}$ also observed a greater risk for anemia related to dapsone in female patients. In the present study, despite of the fact that PB groups were composed of $2 / 3$ of female patients, and that $\mathrm{MB}$ groups by only $1 / 3$ of females, a statistically significant lower hemoglobin level was found only in the MB group treated with 12 doses (MB/R-MDT). The difference observed is likely to be due to the longer treatment. It is important to emphasize that $\mathrm{MB}$ patients treated with 6 doses had a higher length of time to recover when compared to those that received 12 doses. Moreover, it is important to emphasize the known fact that apparently healthier females tend to present more anemia than males. ${ }^{21}$

The MCHC was the only parameter without significant alterations in the different arms of the study. A month after the first MDT dose, the MCV and MCH increased in all groups. This change was more evident in the $\mathrm{PB} / \mathrm{U}-\mathrm{MDT}$ group and remained higher during the whole treatment; similar results were observed in $\mathrm{MB} / \mathrm{R}$ MDT patients receiving 12 doses. Singh et al ${ }^{4}$ also reported significant increase in MCV and $\mathrm{MCH}$ parameters but different from our results, reported statistically significant decrease of $\mathrm{MCHC}$ in patients receiving MDT.

In spite of the decrease in the blood hemoglobin levels, probably caused by dapsone, only $24(2.8 \%)$ of our patients had to stop MDT. There are retrospective studies reporting higher frequen- cies of low blood hemoglobin levels related to dapsone: $18.7 \%$ and $24.2 \%$ in two different reports; these patients had to receive alternative treatments. ${ }^{5,9}$ A lower incidence - $9(11 \%)$ patients, was reported in a prospective study with 79 patients. ${ }^{4}$ The intense monitoring of the patients and the immediate therapeutic action as established in our protocol, avoided the interruption of the treatment.

\section{CONCLUSIONS}

Skin pigmentation and xerosis were the most frequent adverse-effects associated with MDT. The similarities of $\mathrm{AE}$ in the four arms of the study points to the feasibility of a six-month uniform regimen.

Hematological changes were observed in the four arms of the study; it was detected one month after the first dose of MDT and remained changed during the whole treatment. The risk of anemia was greater in females, and MB patients treated with 12 doses were affected with a higher frequency.

The shorter duration of U-MDT treatment minimizes the $\mathrm{AE}$, namely those secondary to dapsone.

Monthly monitoring enables early detection of AE, facilitating prompt interventions and unnecessary interruption or change of treatment.

Based on the present data it is possible to conclude that U-MDT is safe and might be implemented at all levels of leprosy control program. 


\section{REFERENCES}

1. Who.int [Internet]. World Health Organization (WHO). Chemotherapy of Leprosy for Control Programmes - Report of a WHO Study Group. WHO: Geneva; 1982. [cited 2017 Dec 18]. Available from: http://apps. who.int/iris/handle/10665/38984.

2. World Health Organization (WHO). Multidrug Therapy against Leprosy Development and Implementation over the Past 25 Years. Geneva: WHO; 2004.

3. Ekambaram V, Rao MK. Changing picture of leprosy in North Arcot District, Tamil Nadu after M.D.T. Indian J Lepr. 1989;61:31-43.

4. Singh H, Nel B, Dey V, Tiwari P, Dulhani N. Adverse effects of multi-drug therapy in leprosy, a two years' experience (2006-2008) in tertiary health care centre in the tribal region of Chhattisgarh State (Bastar, Jagdalpur). Lepr Rev. 2011;82:17-24.

5. Deps PD, Nasser S, Guerra P, Simon M, Birshner Rde C, Rodrigues LC. Adverse effects from multi-drug therapy in leprosy: a Brazilian study. Lepr Rev. 2007;78:216-22.

6. Dupnik KM, Cardoso FJ, De Macêdo AL, De Sousa IL, Leite RC, Jerônimo SM, et al. Intolerance to leprosy multi-drug therapy: more common in women? Lepr Rev. 2013:84:209-18.

7. Brasil MT, Opromolla DV, Marzliak ML, Nogueira W. Results of a surveillance system for adverse effects in leprosy's WHO/MDT. Int J Lepr Other Mycobact Dis. 1996;64:97-104.

8. Cunha Mda G, Schettini AP, Pereira ES, Pedrosa VL, Cruz RC, Sadahiro M. Regarding Brasil, et al.'s adverse effects in leprosy WHO/MDT and paramedic role in leprosy control program. Int J Lepr Other Mycobact Dis. 1997;65:257-9.

9. Kubota MR, Brancini VCL, Gouveia AS, et al. Efeitos Adversos da poliquimioterapia para hanseníase : Utilização de doses alternativas. Hansen Int. 2014;39:8-21.

10. World Health Organization (WHO). WHO Technical Report Series: WHO Expert Committee on Leprosy - Seventh Report. Vol 874. Geneva: WHO; 1998.

11. Cellona RV, Balagon MF, dela Cruz EC, Burgos JA, Abalos RM, Walsh GP, et al. Long-term efficacy of 2 year WHO multiple drug therapy (MDT) in multibacillary (MB) leprosy patients. Int J Lepr Other Mycobact Dis. 2003;71:308-19.
12. Penna GO. Leprosy: the need to employ evidence-based medicine in control policies around the world. Lepr Rev. 2011;82:210-2.

13. World Health Organization (WHO). Report on Third Meeting of the WHO Technical Advisory Group on Elimination of Leprosy. Brasilia, 1 and 2 February 2002. WHO. 2002:1-19.

14. Kroger A, Pannikar V, Htoon MT, Jamesh A, Katoch K, Krishnamurthy P, et al. International open trial of uniform multi-drug therapy regimen for 6 months for all types of leprosy patients: Rationale, design and preliminary results. Trop Med Int Health. 2008;13:594-602.

15. Rao PN, Suneetha S, Pratap DV. Comparative study of uniform-MDT and WHO MDT in Pauci and Multi bacillary leprosy patients over 24 months of observation. Lepr Rev. 2009:80:143-55.

16. Butlin CR, Pahan D, Maug AKJ, Withington PN, Alam K, Salim AH. Outcome of 6 months MBMDT in MB patients in Bangladesh-preliminary results Introduction/ Background. Lepr Rev. 2016;87:171-82.

17. Penna GO, Pontes MA, Cruz R, Gonçalves Hde S, Penna ML, Bührer-Sékula S. A clinical trial for uniform multidrug therapy for leprosy patients in Brazil: Rationale and design. Mem Inst Oswaldo Cruz. 2012;107:22-7.

18. Hoffbrand AV, Moss PAH. Fundamentos em Hematologia. 6. ed. Porto Alegre: Artmed; 2013. p.425.

19. Ctep.cancer.gov [Internet]. National Institute of Cancer. Common Terminology Criteria for Adverse Events (CTCAE). Version 3.0, DCTD, NCI, NIH, DHHS. March 31, 2003. Publish Date: August 9, 2006. [cited 2017 Dec 18]. Available from: http://ctep.cancer.gov

20. Ferreira IP, Buhrer-Sékula S, De Oliveira MR, Gonçalves Hde S, Pontes MA, Penna ML, et al. Patient profile and treatment satisfaction of Brazilian leprosy patients in a clinical trial of uniform six-month multidrug therapy (U-MDT/CT-BR). Lepr Rev. 2014;85:267-74.

21. McLennan WJ, Andrews GR, Macleod C, Caird Fl.. Anaemia in the elderly. Q J Med. 1973:42:1-13.

$\begin{array}{ll}\text { Rossilene Conceição da Silva Cruz } & \text { (D) ORCID 0000-0002-7735-7687 } \\ \text { Samira Bührer-Sékula } & \text { (D) ORCID 0000-0002-5984-7770 } \\ \text { Gerson Oliveira Penna } & \text { (D) ORCID 0000-0001-8967-536X } \\ \text { Maria Elisabete Amaral de Moraes } & \text { (D) ORCID 0000-0002-6826-8930 } \\ \text { Heitor de Sá Gonçalves } & \text { (D) ORCID 0000-0001-9062-2580 }\end{array}$

$\begin{array}{ll}\text { Mariane Martins de Araújo Stefani } & \text { (D) ORCID } 0000-0001-7874-4315 \\ \text { Maria Lúcia Fernandes Penna } & \text { (iD) ORCID } 0000-0003-0371-8037 \\ \text { Maria Araci de Andrade Pontes } & \text { (iD) ORCID } 0000-0002-7292-8452 \\ \text { Sinésio Talhari } & \text { (iD) ORCID } 0000-0001-9753-6706\end{array}$

How to cite this article: Cruz RCS, Bührer-Sékula S, Penna GO, Moraes MEA, Gonçalves HS, Stefani MMA, Penna MLF, Pontes MAA, Talhari S. Clinical trial for uniform multidrug therapy for leprosy patients in Brazil (U-MDT/CT-BR): adverse effects approach. An Bras Dermatol. 2018;93(3):377-84. 